\title{
Identification of Polycomb Repressive Complex1, Trithorax group genes and their simultaneous expression with WUSCHEL, WUSCHEL-related Homeobox5 and SHOOT MERISTEMLESS during the induction phase of somatic embryogenesis in Medicago truncatula Gaertn.
}

\author{
Anna Orłowska ${ }^{1} \cdot$ Ewa Kępczyńska $^{1}$
}

Received: 22 January 2018 / Accepted: 13 May 2018 / Published online: 19 May 2018

(c) The Author(s) 2018

\begin{abstract}
The Polycomb Repressive Complex1 (LHP1, RING1, BMI1, EMF1, VRN1) and Trithorax group (ATX2, ATX3.1, ATX3.2, ATX5-like) genes, were for the first time identified in the genome of Medicago truncatula. Their expression, along with that of the earlier-identified MtWUS, MtWOX5 and MtSTM, was investigated during the induction phase (IP). During the 21-day-long IP, all the genes mentioned, except for MtSTM, were expressed in non-embryogenic (M9) and embryogenic (M9-10a) genotype of M. truncatula. The lower expression level of all the PRC1 genes in primary explants of the M9-10a line compared to their expression in the M9 line probably triggers processes leading to the formation of embryogenic cells. In addition, the higher-from the second day of the induction phase-expression of the ATX2, ATX3.1 and ATX3.2 genes from the $\operatorname{Tr} x G$ in cells of the embryogenic line, compared to the expression in the non-embryogenic one, suggests their involvement in acquisition of cell competence for embryogenesis. Among the three tested genes known for their involvement in organization of the meristematic centers in zygotic embryos (MtWUS, MtWOX5, MtSTM), two (MtWUS and MtWOX5) can serve as markers of cell dedifferentiation in leaf explants of both lines. Moreover, MtSTM may mark embryogenic cells, since its expression was registered only in the embryogenic line. The study provides new data regarding involvement of the PRCl and TrxG genes during prime events of the SE and sheds new light on the involvement of MtWUS, MtWOX5 and $M t S T M$ in the process.
\end{abstract}

Keywords Somatic embryogenesis $\cdot$ Fabaceae $\cdot$ Epigenetic and transcriptional regulation $\cdot$ Polycomb $\cdot$ Trithorax $\cdot W U S$. WOX5 - STM

$\begin{array}{ll}\text { Abbreviations } \\ \text { ATX1-5 } & \text { Arabidopsis Trithorax 1-5 } \\ \text { EMF1 } & \text { Embryonic Flower1 } \\ \text { IP } & \text { Induction phase } \\ \text { LHP1 } & \text { Like Heterochromatin1 } \\ \text { PRC1 } & \text { Polycomb Repressive Complex1 } \\ \text { STM } & \text { Shoot Meristemless } \\ \text { TrxG } & \text { Trithorax group } \\ \text { VRN1 } & \text { Vernalization1 }\end{array}$

Communicated by Sergio J. Ochatt.

Ewa Kępczyńska

ekepcz@univ.szczecin.pl

1 Department of Plant Biotechnology, Faculty of Biology, University of Szczecin, Wąska 13, 71-415 Szczecin, Poland
WOX5 Wuschel-related Homeobox5

WUS Wuschel

\section{Introduction}

Somatic embryogenesis (SE) is a complex biological process in which somatic plant cells acquire the capacity to transform into embryos, following which a complete plant can be regenerated. The process can be initiated in vitro from sporophytic (Zimmerman 1993; Fehér et al. 2003) or gametophytic cells (Yang and Zhou 1982; Reynolds 1997). Under specific in vitro conditions, the embryogenic pathway may be directly (direct somatic embryogenesis, DSE) or indirectly (indirect somatic embryogenesis, ISE) initiated in differentiated explant's cells through the callus/PEM (proembryogenic mass) origination. Callus 
formation, i.e., the emergence of cell masses showing varying degree of dedifferentiation of explants, mainly in the presence of an auxinic herbicide, 2,4-dichlorophenoxyacetic acid (2,4-D), and cytokinins, is associated with significant changes within the cells. Such reorganization in cell identities and growth patterns is accompanied by changes in the expression of numerous genes on transcriptional and epigenetic levels. In the past decades, a considerable effort was expended to identify genes, which expression patterns changed during SE, mainly in Arabidopsis. Several marker genes have been reported that are able to distinguish between embryogenic and non-embryogenic cells such as SOMATIC EMBRYOGENESIS RECEPTOR KINASE (SERK; Schmidt et al. 1997; Nolan et al. 2009), LEAFY COTYLEDON1 (LEC1; Lotan et al. 1998; Ledwoń and Gaj 2011; Orłowska et al. 2017), LEAFY COTYLEDON1-LIKE (L1L; Orłowska et al. 2017), FUSCA3 (FUS3; Luerssen et al. 1998; Ledwoń and Gaj 2011) and $B A B Y$ BOOM (BBM; Igielski and Kępczyńska 2017).

The dynamic and rapidly induced somatic explant cell's response to stimuli (hormones and stress), including the expression of transcriptional factors, involves epigenetic modifications of chromatin. Modification of the chromatin structure can change the expression of many genes. SE is known to be controlled by "three pillars of epigenetics": RNA mechanisms, DNA methylation and histone modifications (Smertenko and Bozhkov 2014; De-la-Peña et al. 2015). Histone modifications which lead to epigenetic changes affecting gene expression are catalyzed by the Polycomb group (PcG) and Trithorax group (TrxG) proteins. Both groups act as critical modulators of plant development and are essential for maintaining cells identity, their fate determination and differentiation (Hennig and Derkacheva 2009; Bratzel et al. 2010; de la Paz Sanchez et al. 2015). PcG and TrxG regulate transcriptional repression and activation of genes, respectively, through the activity of histone lysine methyltransferase (HKMT) (Schuettengruber et al. 2011). In plants, PcG acts as two conservative protein complexes: the Polycomb Repressive Complex1 (PRC1) and PRC2. The well-characterized PRC2 catalyzes trimethylation of histone $\mathrm{H} 3$ lysine 27 (H3K27me3), while PRC1 binds to H3 K27me3 marks and ubiquitinates of histone H2A lysine 119 (H2AK27ub), which keeps the chromatin in a packed state (He et al. 2012). Currently, it is thought that the Arabidopsis PRC1 complex comprises five subunits: the H3K27me3 reader protein-LIKE HETEROCHROMATIN PROTEIN1 (LHP1), also known as TERMINAL FLOWER2 (TFL2); the H2Aub1 writer proteins-RING1a-b and BMI1a-c proteins; and one non-sequence specific DNA-binding protein-EMBRYONIC FLOWER1 (EMF1) or VERNALIZATION1 (VRN1) (Turck et al. 2007; Calonje et al. 2008; Bratzel et al. 2010; Calonje 2014). In contrast to the
Polycomb proteins, TrxG act as positive regulators of gene expression by catalyzing of histone $\mathrm{H} 3$ lysine 4 trimethylation (H3K4me3) of the target genes (Schuettengruber et al. 2011). Five ARABIDOPSIS TRITHORAX1-5 (ATX1-5) and two ATX-RELATED (ATXR3 and ATXR7) proteins with histone methylotransferases activity in Arabidopsis are known (Springer et al. 2003; Ng et al. 2007).

Information on the contribution of the PRC1 and TrxG proteins to SE regulation is very scarce and concerns only mutants with a partial or complete inhibition of several genes belonging to the two groups. The loss in expression of the gene encoding the H2Aub1 writer proteins results in many disorders of the development program. Atringla Atring $1 b$ and Atbmila Atbmilb, the Arabidopsis double mutants, showed derepression of embryogenic traits in the somatic tissue (Bratzel et al. 2010; Chen et al. 2010). Plants with a partial or complete inhibition of the BMII expression formed embryo- and callus-like structures from cotyledons, apex and leaves. Both RING1 and BMI1 are involved in regulation of embryogenic and stem cell development and are required to maintain somatic cells in the differentiation state (Yang et al. 2013). Mutations in the ATXI gene cause abnormal flower development and disorders in the formation of the callus tissue from leaf explants, but are not involved in the embryogenesis (Alvarez-Venegas et al. 2003; He et al. 2012). To date, expression of genes encoding PRC1 and TrxG components during SE has not been described. Moreover, there have been no studies in which expression of the three genes [SHOOT MERISTEMLESS (STM); WUSCHEL (WUS); WUSCHEL-related homeobox5 (WOX5)] would be compared at the same time during Medicago truncatula induction phase, comparisons effected so far concerned only WUS and WOX5 (Zuo et al. 2002; Scofield et al. 2014).

WUS, WOX5 and STM encode proteins representing homeodomain transcription factors which participate in the organization of the meristematic centers (Endrizzi et al. 1996; Sarkar et al. 2007). During zygotic embryogenesis expression of WUS is not only related to its role in the apical meristem, but also in early ovule development of Arabidopsis thaliana and M. truncatula (Groß-Hardt et al. 2002; Kurdyukov et al. 2014). Zuo et al. (2002) showed that WUS plays a critical role in the maintenance of the embryogenic cell identity during SE. Ectopic expression of AtWUS in Coffea canephora induces callus formation and causes an increase in somatic embryos production (Arroyo-Herrera et al. 2008). An identical ectopic expression in Gossypium hirsutum WUS promotes the vegetative-to-embryogenic transition (Zheng et al. 2014). Chen et al. (2009) investigated WUS and additionally WOX5 and found WUS expression to be essential for $M$. truncatula callus growth and somatic embryo induction. STM controls expression of the KNOX genes and, similarly to WUS, is responsible for maintaining the stem cell function in the apical meristem (Scofield et al. 
2014). STM is expressed in all the cells of the shoot apical meristem in zygotic embryos of Arabidopsis (Long et al. 1996). It was also up-regulated in early somatic embryos of Glycine max L. (Ma et al. 1994). The ectopic expression of Brassica napus STM initiates SE (Elthiti et al. 2010).

To date, identification of genes encoding the PRC1 complex and Trithorax group components and their expression during the induction phase (IP) in M. truncatula SE has not been reported. Moreover, to our best knowledge, no studies have examined the dynamics of gene expression, during the SE induction, of transcription factors WUS and WOX5 together with STM, which are known regulators of the in vivo stem cell maintenance. So, the present study was carried out in order to: (i) identify genes coding proteins of the PRC1 complex (LHP1, RING1, BMI1, EMF1, VRN1), TrxG (ATX2, ATX3.1, ATX3.2, ATX5-like); (ii) explore their expression during the IP in the non-embryogenic (M9) and embryogenic (M9-10a) line of M. truncatula; and (iii) compare gene expression of WUS, WOX5 and STM in the two lines mentioned. The results should shed new light on the involvement of epigenetic regulation and homeodomain transcription factors in the initiation of the transition of somatic leaf cells to embryogenic ones in M. truncatula somatic embryogenesis.

\section{Materials and methods}

\section{Tissue culture protocol}

Callus tissue samples were collected from two $M$. truncatula Gaertn. lines, i.e., non-embryogenic (M9) and embryogenic (M9-10a) (Fig. 1), according to the protocol described previously (Orłowska et al. 2017).

\section{Sequence analysis and construction of phylogenetic trees}

The amino acid sequences of $A$. thaliana $P R C 1$ and $\operatorname{Tr} x G$ genes were obtained from the TAIR database (http://www. arabidopsis.org/) and used to BLAST search against the M. truncatula genome database (http://www.medicagoge nome.org/). Specific domain locations were confirmed in the InterPro database. The homology of protein sequences was checked by the sequence alignments analysis using the ClustalW of Geneious 6.1 software (http://www.geneious. com, Kearse et al. 2012). The phylogenetic trees were constructed with the Neighbor-Joining method.

\section{Molecular analysis}

Total RNA was extracted from the plant material at five time points (induction day 0, 2, 7, 14 and 21) using Direct-zol ${ }^{\mathrm{TM}}$ RNA-MiniPrep Kit (ZymoResearch), as described previously (Orłowska et al. 2017). qPCR was performed with the $5 \times$ HOT FIREPol ${ }^{\circledR}$ EvaGreen ${ }^{\circledR}$ qPCR Mix Plus (ROX) (Solis BioDyne) using the STEP ONE Real-time PCR System (LifeTechnologies), following the manufacturer's instructions. All primer sequences for qPCR are shown in Table 1. The relative expression level was normalized to ACTIN2 using the $2^{-\Delta \Delta \mathrm{Ct}}$ method (Livak and Schmittgen 2001). GenEX software (MultiD Analyses AB, Sweden) was used to analyzed data. Three biological replicates of each time point and three technical ones were analyzed.

All the experiments were carried out in triplicate. Changes in gene expression among days of induction phase were analyzed using the GenEX software (MultiD Analyses $A B$, Sweden). The results are expressed as mean \pm SD. Statistical analyses were performed using the ANOVA. Differences between the mean values were considered to be significant at $\mathrm{p}<0.01$ or $\mathrm{p}<0.05$.

\section{Results}

\section{Identification and phylogeny of Medicago truncatula genes}

Before the gene expression experiments the sequence alignment analysis of proteins encoded by $P R C 1$ and $\operatorname{Tr} x G$ genes
Fig. 1 Callus tissue development on the SH medium supplemented with $0.5 \mu \mathrm{M} 2,4-\mathrm{D}$ and $1 \mu \mathrm{M}$ zeatin (21 days) from primary leaf explants of $M$. truncatula non-embryogenic (M9) and embryogenic (M9-10a) lines, and somatic embryo formation on the MS medium (14 days) (black dots on the time line mark sample collection events)

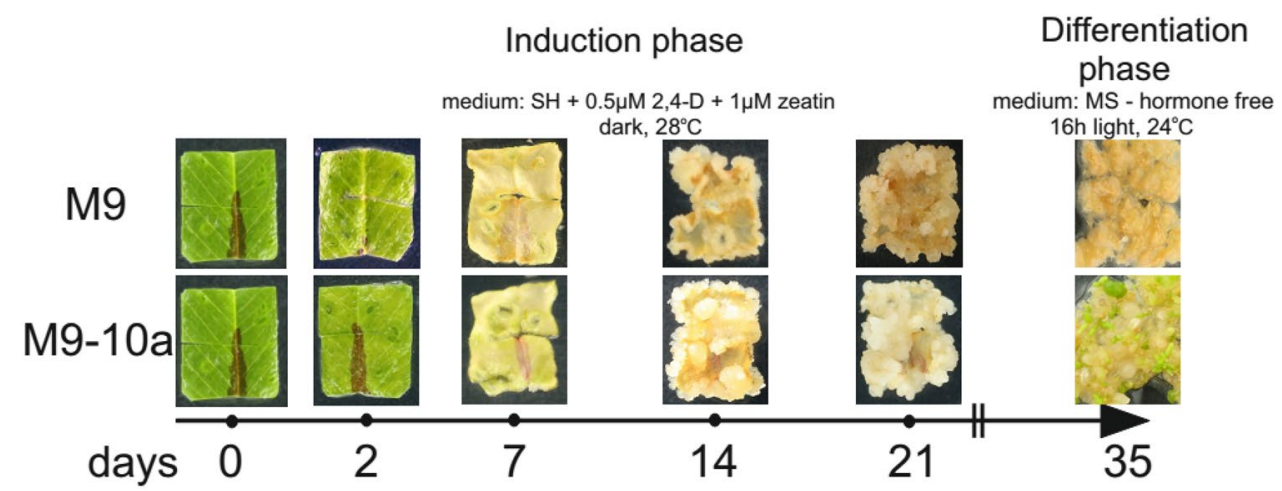


Table 1 Description of the analyzed genes and their primer sequence

\begin{tabular}{|c|c|c|c|}
\hline Gene name short & $\begin{array}{l}\text { Accession number } \\
\text { nucleotide/protein }\end{array}$ & Gene symbol & Primer sequence \\
\hline \multirow[t]{2}{*}{ LHP1/TFL2 } & XM_003604031.1 & MTR_3g118260 & F: CTTCACCGCAGAGGAACCAA \\
\hline & XP_003604079.1 & & R: AGGAGCAGTGGAGCCAAGTG \\
\hline \multirow[t]{2}{*}{ RING1 } & XM_013601530.1 & MTR_4g088520 & F: AGCAACAGCAGCGGCTTTTAT \\
\hline & XP_013456984.1 & & R: TACCTGAAAGTCGCCAGCATT \\
\hline \multirow[t]{2}{*}{ BMII } & XM_013595075.1 & MTR_7g115240 & F: CCAAATCGGAGCCACCTACAT \\
\hline & XP_013450529.1 & & R: TGTTTCCCCCCTTGTCATCTC \\
\hline \multirow[t]{2}{*}{$E M F 1$} & XM_013606538.1 & MTR_3g110082 & F: CAAGGCCAATGGTACCCTGAA \\
\hline & XP_013461992.1 & & R: CCGAAGGTTGGAGAGCCAAT \\
\hline \multirow[t]{2}{*}{ VRN1 } & XM_013611264.1 & MTR_1g034240 & F: CATCAAGAAAGAAGCGGAAATCA \\
\hline & XP_013466718.1 & & R: GCAGACGCACTTTCATAGAATCG \\
\hline \multirow[t]{2}{*}{ ATX2 } & XM_013592324.1 & MTR_7g021365 & F: TGGTGGGTGCTGGAACGTATA \\
\hline & XP_013447778.1 & & R: TGGTGCGCAGGAATGATTAAT \\
\hline \multirow[t]{2}{*}{ ATX3.1 } & XM_013595146.1 & MTR_7g117355 & F: GGGAACGGCATACAGGTTGT \\
\hline & XP_013450600.1 & & R: GGATTGTGTTCCGTGATCCAT \\
\hline \multirow[t]{2}{*}{ ATX3.2 } & XM_013589183.1 & MTR_8g027725 & F: GATTCAAGGAAGCGGACACCTA \\
\hline & XP_013444637.1 & & R: GCGGTAGCATTGTGCTTTTCA \\
\hline \multirow[t]{2}{*}{ ATX5-like } & XM_003588480.2 & MTR_1g008230 & F: GAACGCCACACAGGTTCCAA \\
\hline & XP_003588528.2 & & R: AATCAGCCAACTGTAGCATCCAT \\
\hline \multirow[t]{2}{*}{$W U S$} & XM_003612110.1 & MTR_5g021930 & F: TTCATCTGCTGGGCTTTCTTC \\
\hline & XP_003612158.1 & & R: TGCTGCTACATCCAGCTGAAAT \\
\hline \multirow[t]{2}{*}{ WOX5 } & XM_003616533.2 & MTR_5g081990 & F: ACTGGCACAAAGTGTGGTCGTT \\
\hline & XP_003616581.1 & & R: TTTGATCAGTGCTTGGAGTTCTG \\
\hline \multirow[t]{2}{*}{$S T M$} & XM_013607446.1 & MTR_2g024390 & F: GCCTTACCCATCTTGAATCACAGA \\
\hline & XP_013462900.1 & & R: CCAGTGCCGTTTCCTTTGATTA \\
\hline
\end{tabular}

were carried out. STM (Mt-Knox-1), WUS and WOX5 studied in this work were earlier identified in M. truncatula by Koltai et al. (2001) and Chen et al. (2009), respectively.

Analysis of PRC1 and $\operatorname{Trx} G$ proteins was based on amino acid sequence similarity and organization of protein domains. Amino acid analysis of LHP1 showed all of the Fabaceae (M. truncatula, Cicer arietinum, Glycine max and Phaseolus vulgaris) LHP1 belonged to the same clade (Fig. 2a). The domain organization analysis showed LHP1 of $A$. thaliana and $M$. truncatula, to possess two characteristic domains: chromodomain and chromo shadow domain (Fig. 3). The BLAST analysis of RING1 and BMI1 (also described as DRIP2) revealed products of just one gene in Medicago, as opposed to two and three genes, respectively, in the Arabidopsis genome (Fig. 2b, c). All the RING1 and BMI1 paralogue proteins of Arabidopsis were grouped in one clade. The sequence analysis revealed the RING/FYVE/ PHD-type Zinc finger domain to be located on N-tails of $A$. thaliana and M. truncatula (Fig. 3). The last two components of the PRC1 complex tested were EMF1 and VRN1 (Fig. 2d, e). Analysis of the EMF1 protein showed no characteristic domain in both plants (Fig. 3). In contrast, the VRN1 protein of $A$. thaliana and $M$. truncatula revealed a
DNA-binding pseudo-barrel domain (B3 domain) located at the $\mathrm{N}$ - and $\mathrm{C}$-terminus.

The amino acid analysis of ATX proteins from the Trithorax group showed the presence of two fabacean ATX3 proteins, compared to just one in A. thaliana and B. napus (Fig. 4). Both Fabaceae ATX3 formed a sister group to each other. The sequence analysis of the A. thaliana and $M$. truncatula ATX showed the presence of a few domains in all the proteins analyzed: PWWP, Zinc finger, SET with ending Post-SET domain (Fig. 3). The ATX2 protein has additionally poorly characterized FY-rich domains (N-terminal and C-terminal), the ATX3 of A. thaliana and ATX3.2 of $M$. truncatula showing a SAND-like domain, absent in $M t$ ATX3.1.

The phylogenetic trees of the PRC1 complex and TrxG components (Figs. 2, 4) showed all of the M. truncatula proteins analyzed to form one clade alongside other PRC1 and $\operatorname{Trx} G$ fabacean proteins, respectively.

\section{Expression of the PRC1 and TrxG genes}

The expression profile analysis of all the PRC1 genes shows their transcripts level in primary explants to be about three times higher in the non-embryogenic (M9) than in the 
Fig. 2 Unrooted phylogenetic trees based on deduced amino acid sequences of Medicago truncatula LHP1 (a), RING1 (b), BMI1 (c), EMF1 (d) and VRN1 (e). At, Arabidopsis thaliana; $\mathrm{Bn}$, Brassica napus; $\mathrm{Ca}$, Cicer arietinum; $\mathrm{Gm}$, Glycine max; Mt, Medicago truncatula; $\mathrm{Nt}$, Nicotiana tomentosiformis; Tc, Theobroma cacao; $\mathrm{Pv}$, Phaseolus vulgaris; S1, Solanum lycopersicum
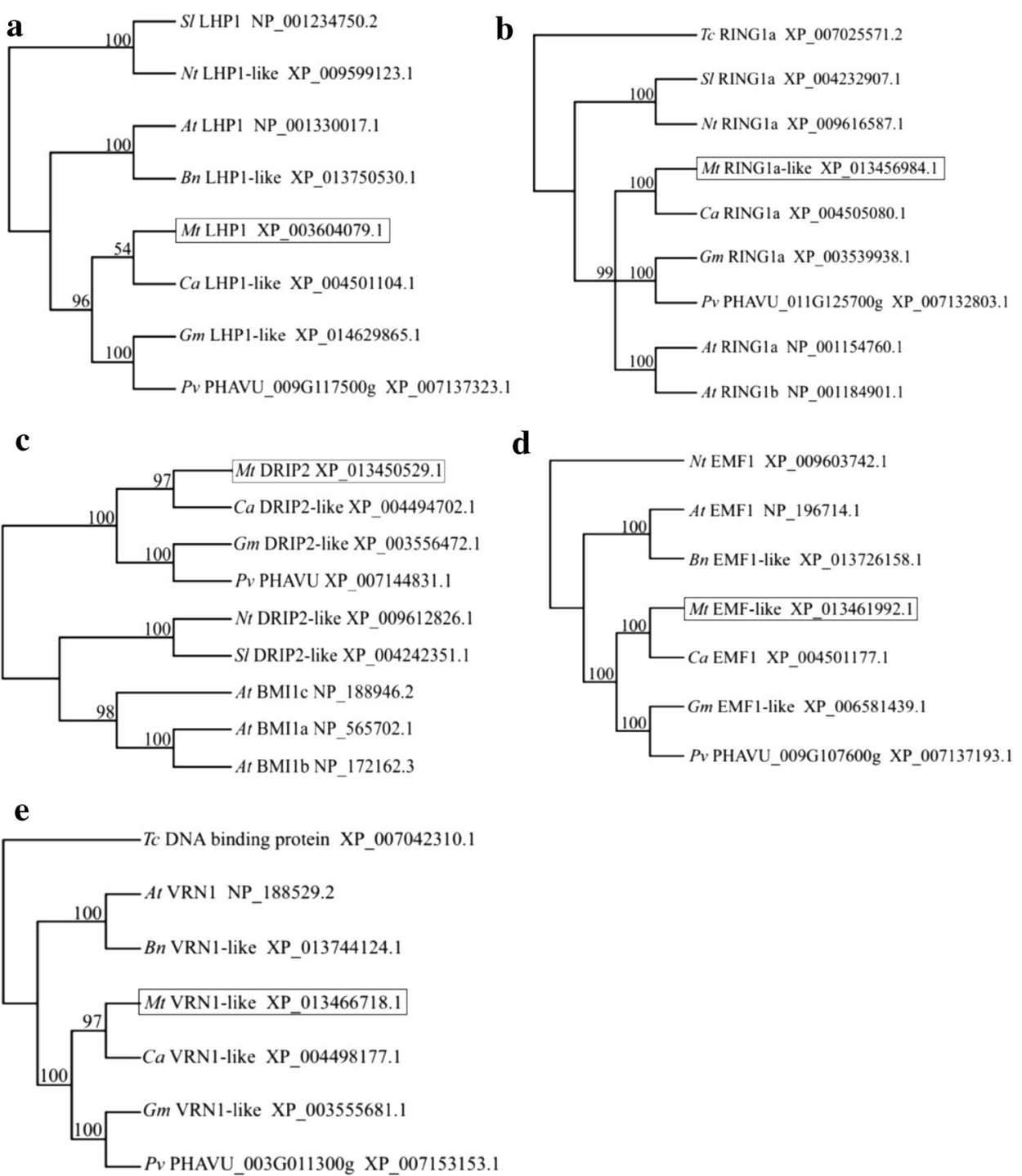

embryogenic (M9-10a) line; in subsequent days, the level varied depending on the gene being analyzed (Fig. 5). The LHP 1 gene expression profile differed in both lines during the first 7 days of the IP, the level being lower in the embryogenic line than in the non-embryogenic one (Fig. 5a). On day 7 , the expression reached an identical level in the two lines and did not change to the end of the IP. The expression profile of M. truncatula RING1 was similar to that observed in the $L H P 1$ gene (Fig. 5b). In primary explants, the expression in the M9 tissue was 3.5 times higher that in the embryogenic one, to reach a similar level on day 7 and to remain stable until termination of the SE induction phase. A different expression pattern was observed in BMII (Fig. 5c). The analysis revealed that in both lines the transcript level reached nearly the same value on day 2 and then increased until day 14, the increase being about 7- and three-fold in the M9-10a and M9 lines, respectively. The transcript level of $E M F 1$ was almost the same in both lines until the end of
IP (Fig. 5d). Of all the PRC1 complex genes analyzed, the largest differences between the two lines in the expression were found in the VRNI gene (Fig. 5e). Its expression in M9 line was similar to that in primary explants during the entire IP. However, the transcript level of the VRN1 gene in the embryogenic line increased gradually in subsequent days of induction to reach the highest value on day 21 when it was almost 7 times higher compared to that in the primary explant (day 0). In summary, as a massive callus tissue was gradually developing in the embryogenic line (Fig. 1), expression of only one of all genes analyzed from this complex, $V R N 1$, also increased.

Since epigenetic factors, such as the Trithorax group, are involved in different developmental processes in Arabidopsis, it was interesting to study if the ATX2, ATX3.1, ATX3.2 and ATX5-like genes, are putatively involved in the transition of cells from somatic to embryogenic once in M. truncatula (Fig. 6). In primary explants, the expression of the ATX2 

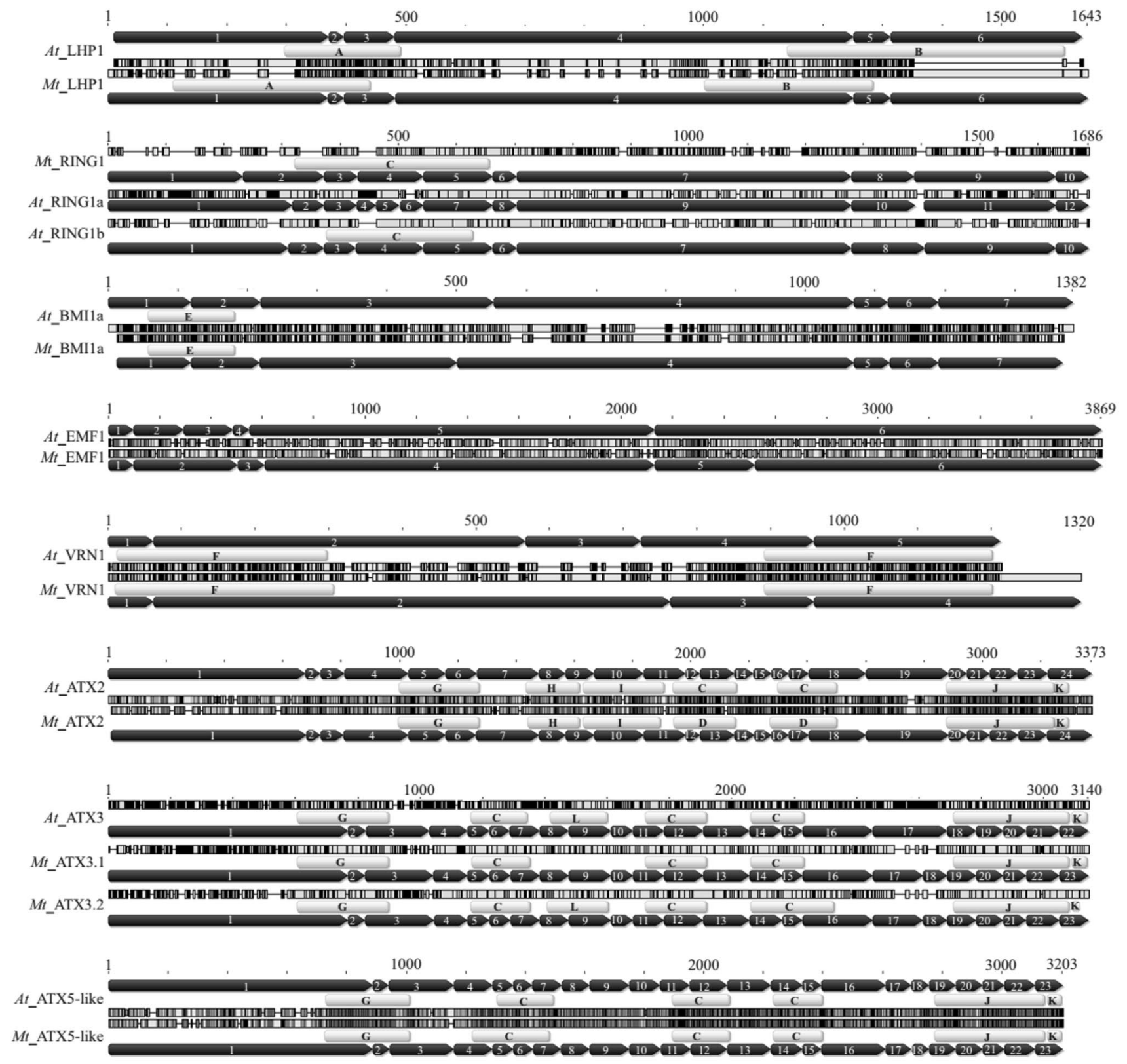
Legend:
A - Chromo domain-like
G - PWWP domain

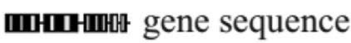
B - Chromo shadow domain
H - FY-rich, N-terminal
A.L domain
C - Zinc finger, RING/FYVE/PHD-type
I - FY-rich, C-terminal
1.24 exon
D - Zinc finger, FYVE/PHD-type
J - SET domain
E - Zinc finger, C3H4 type (RING finger)
K - Post-SET domain
F - DNA-binding pseudobarrel domain
L - SAND domin-like

Fig. 3 A schematic view of the domain location of Polycomb Repressive Complex1 and Trithorax group proteins of Arabidopsis thaliana (At) and Medicago truncatula $(\mathrm{Mt})$

in the non-embryogenic line surpassed that observed in the embryogenic line (Fig. 6a). Its expression in the non-embryogenic line decreased after two days and, until the end of IP, remained on the same level. The primary explants of the
M9-10a line showed the lowest ATX2 expression; it almost doubled during the first 7 days and was unchanged to the end of IP. The ATX3.1 expression observed in primary explants of the M9 and M9-10a lines on day 2 was similar (Fig. 6b). 


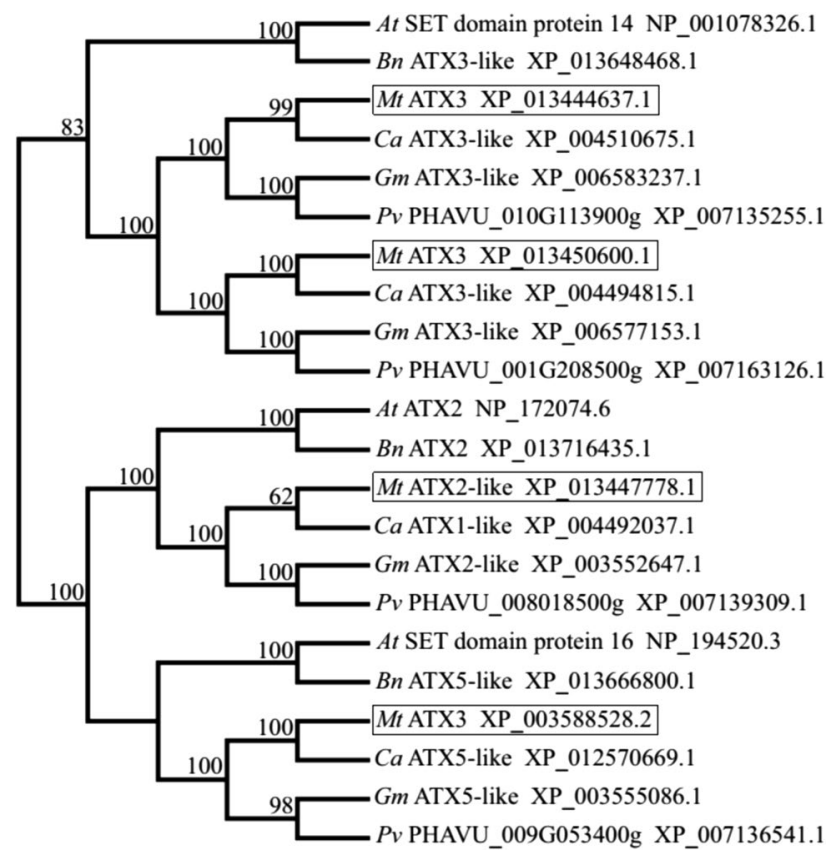

Fig. 4 Unrooted phylogenetic tree based on deduced amino acid sequences of M. truncatula ATX2, ATX3.1, ATX3.2 and ATX5-like. At, Arabidopsis thaliana; Bn, Brassica napus; Ca, Cicer arietinum; $\mathrm{Gm}$, Glycine max; Mt, Medicago truncatula; $\mathrm{Pv}$, Phaseolus vulgaris

However, expression of the gene in the embryogenic line increased significantly between days 2 and 7 of the induction phase, and gradually decreased during the next 2 weeks. The expression profile of ATX3.2 differed from the expression of the two genes described above. The highest levels of transcripts were observed in primary explants of both lines; the expression of the gene in the M9 line was higher that in the M9-10a (Fig. 6c). After 2 days, transcript level of the gene in explants of both lines was rapidly reduced (about sixfold) to a similar level. Then, the ATX3.2 expression in the M9-10a increased after 7 days and remained at a similar level until the end of induction. However, in the non-embryogenic line, it gradually decreased in the first and second week of induction, and increased during the third week. ATX5-like was the only gene among the $\operatorname{Tr} x G$ genes studied whose expression profile was almost the same in both lines during the whole IP (Fig. 6d).

\section{Expression of WUS, WOX5 and STM}

As the WUS, WOX5 and STM proteins are known to participate in the organization of the apical meristematic centers in plants, it was very interesting to compare expression of genes encoding these proteins during callus formation from leaves of the both M. truncatula lines.

Both Medicago lines showed nearly identical expression profiles of MtWUS (Fig. 7a). Expression of the gene in the leaf explants (day 0) was on a minimal level, but day 2 revealed the maximum expression; it was more than 150and 120 -fold higher, respectively, than that in the primary explants of both lines. However, after 7 days, the gene's expression in both lines decreased rapidly, and after 2 and 3 weeks it was at the detectability level. The WOX5 expression was not measurable in primary explants of both lines. The

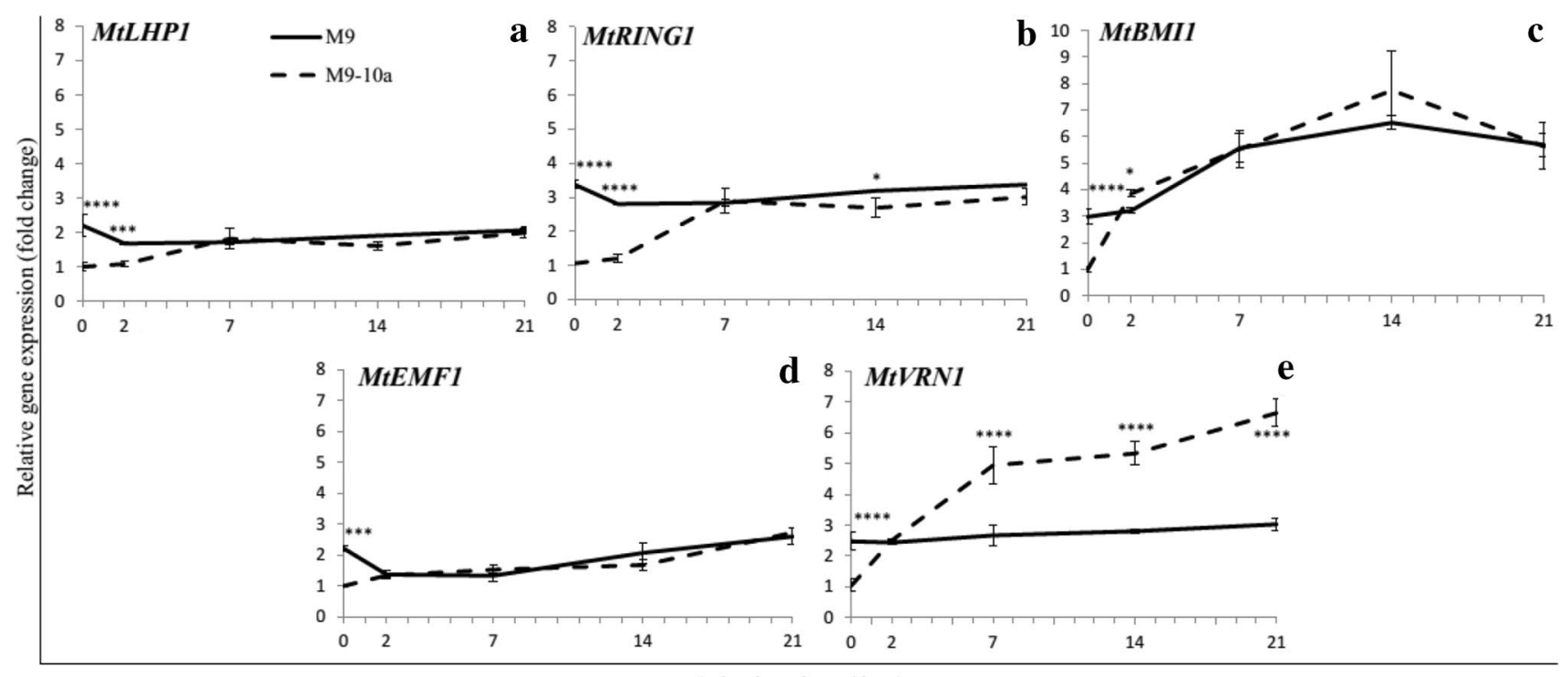

Induction phase (days)

Fig. 5 Relative expression patterns of LHP1 (a), RING1 (b), $B M I 1 / D R I P 2$ (c), EMF1 (d) and VRN1 (e) genes in the leaf explants of Medicago truncatula non-embryogenic (M9) and embryogenic
(M9-10a) lines during the induction phase on the SH medium. Bars show standard deviation. Solid and dotted lines represent the M9 and M9-10a lines, respectively 


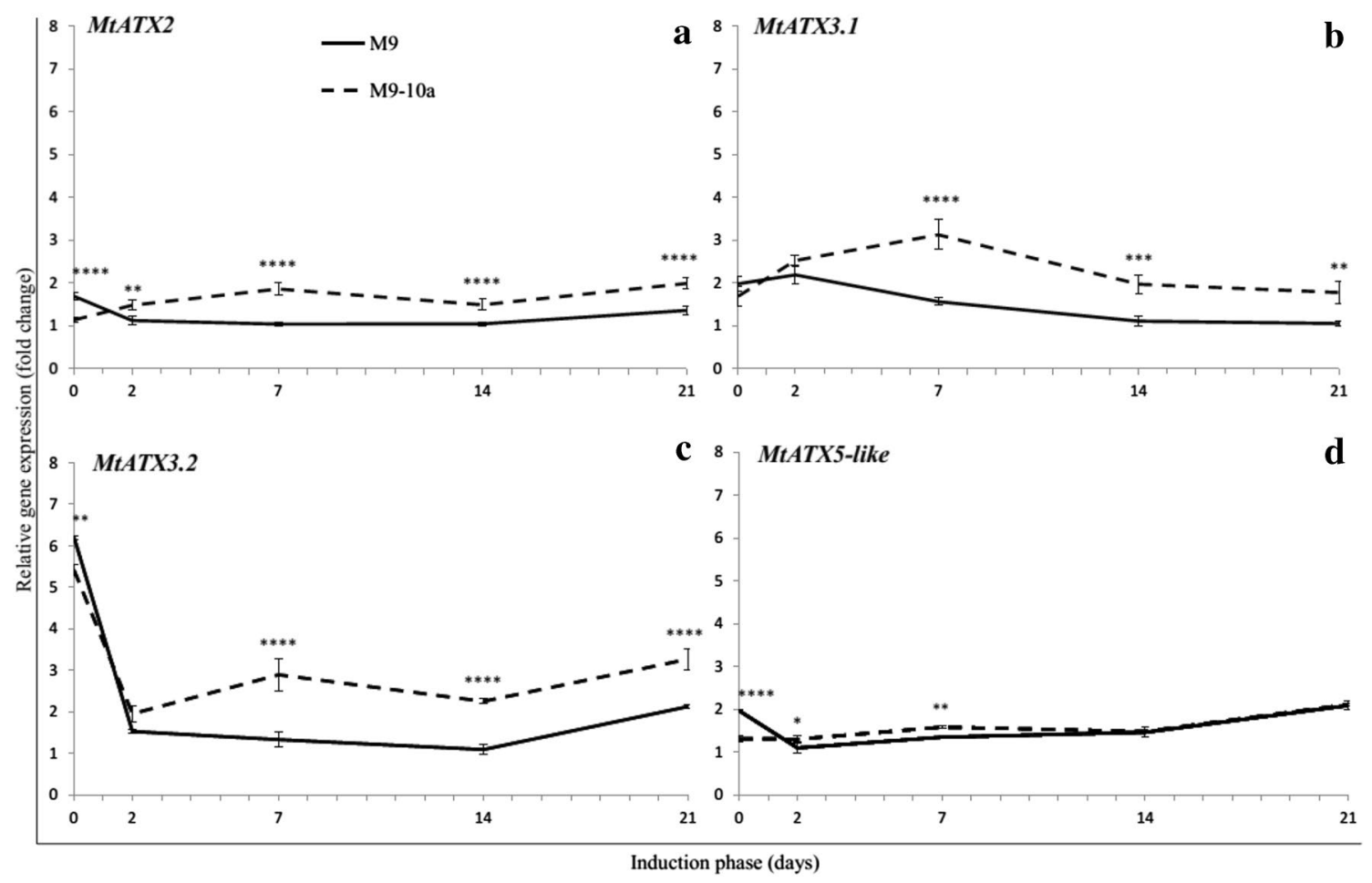

Fig. 6 Relative expression patterns of ATX2 (a), ATX3.1 (b), ATX3.2 (c) and ATX5-like (d) genes in the leaf explants of Medicago truncatula non-embryogenic (M9) and embryogenic (M9-10a) lines during the induction phase on the SH medium. Bars show standard deviation. Solid and dotted lines represent the M9 and M9-10a lines, respectively

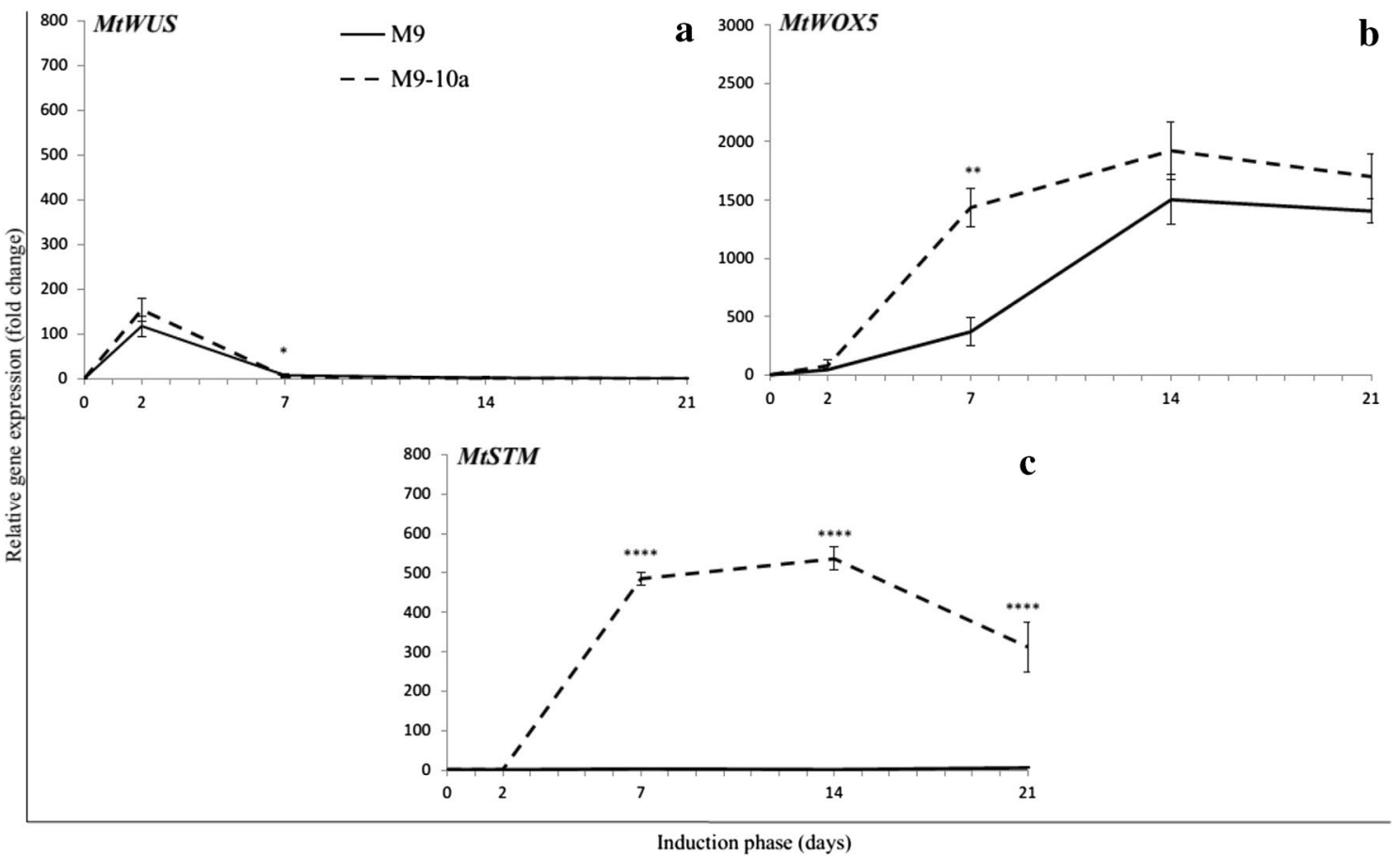

Fig. 7 Relative expression patterns of WUS (a), WOX5 (b) and STM (c) genes in the leaf explants of Medicago truncatula non-embryogenic (M9) and embryogenic (M9-10a) lines during the induction phase on the SH medium. Bars show standard deviation. Solid and dotted lines represent the M9 and M9-10a lines, respectively 
gene showed a drastic upregulation of the expression (from about 81 to $>1900$ fold) between day 2 and day 14 of the IP in the M9-10a line (Fig. 7b). During the subsequent days, the expression remained almost unchanged. In the M9 line, the expression profile of WOX 5 was similar, but its level in all the tissues tested was lower than that in the in embryogenic line. The difference was particularly evident on day 7 of the IP when the transcription level for this gene in both lines was 371 and 1437-fold higher, respectively, than the expression levels in primary explants.

Expression of STM, a class- 1 KNOX gene, unlike that of WUS, was observed only in M9-10a line (Fig. 7c). The expression started between day 2 and 7 , and reached the maximum on day 14 ; it was more than 500 -fold higher, relative to that in primary and 2-day-old explants. The STM expression in all the samples of the M9 line was non-detectable during the entire induction phase.

\section{Discussion}

Although much progress has been made in learning the mechanisms leading to the induction of somatic cell transition to the embryogenic state, this process still remains incompletely understood. During callus formation, differentiated tissues of primary explants must undergo processes eliminating their original characteristics. The necessary processes could be mediated by the balanced activity of the two proteins groups, Trithorax and Polycomb.

Despite that numerous plant genomes were successfully sequenced in the last decades, many genes have not been annotated yet. So this study required identification of genes encoding proteins responsible for the chromatin remodeling in M. truncatula. The amino acid analysis components of the PRC1 complex and TrxG indicate a similarity of those proteins between A. thaliana and the Fabaceae family. Certain single proteins of $A$. thaliana, including LHP1, EMF1, VRN1 and ATX2 are also single in M. truncatula. However, there are some Arabidopsis paralogue proteins, like BMIa-c and RING1a-b, which in M. truncatula are represented by a single protein. The domain analysis of LHP1 in both plants showed them to possess a chromodomain and a chromo shadow domain, required for proper activity of the protein (Hennig and Derkacheva 2009). The PRC1 complex is also built by the RING1 and BMI1 belonging to the RING finger family. The RING/FYVE/PHD-type Zinc finger domain binds DNA and is involved in protein-protein interactions (Takatsuji 1998). The location of the RING domain at the N-terminus of RING1 and BMI1 proteins was also confirmed by Sanchez-Pulido et al. (2008) in A. thaliana, Oryza sativa and Vitis vinifera. In contrast to their analysis, the presence of the RAWUL domain on the C-terminus of these proteins was not confirmed in this study. Similarly to the results of King et al. (2013) for Arabidopsis, the domain analysis of the M. truncatula VRN1 indicated the protein to possess a DNA-binding pseudobarrel domain (B3 domain) located at the $\mathrm{N}$ - and $\mathrm{C}$-terminus.

The phylogenetic analysis of Trithorax group proteins showed that Mt ATX3 occur as two paralogues (ATX3.1 and ATX3.2) similar to only one protein in A. thaliana. Alvarez-Venegas and Avramova (2012) confirmed presence of PWWP domain in the A. thaliana ATX proteins. The presence of FY-rich and SAND domains in Arabidopsis TrxG proteins was confirmed by Chen et al. (2017).

Since the PRC1 and TrxG proteins are also known to participate in switch the vegetative development program to the generative one in Arabidopsis (Alvarez-Venegas et al. 2003) and M. truncatula (Jaudal et al. 2016), it was adequate to check the expression of above mentioned genes during the transition of somatic to embryogenic cells that occurs during early events of induction phase. The lower expression level of the $P R C 1$ genes tested (LHP1, RING1, $B M I 1 a, E M F 1, V R N 1)$ in M. truncatula primary explants of the embryogenic line, compared to their expression in the non-embryogenic line, probably allows the initiation of dedifferentiation processes. Such a phenomenon was observed by Mozgová et al. (2017) with the PRC2 during A. thaliana SE. They showed that partial and transient reduction of the PRC2 activity is enough for 2,4-D-mediated SE. The higher expression of all the $P R C 1$ genes tested in the M. truncatula primary explant non-embryogenic line than in embryogenic line may be responsible for the inhibition of SE induction; it may be related to a different level of chromatin trimethylation and ubiquitination in primary explants. Two of PRC1 complex genes, MtLHPI and MtRING1, could be probably included in the regulation of SE only up to IP day 7. It was reported, that lhpl/tfl2 mutants showed the lack of fertilization and defective seed development (Larsson et al. 1998). In Arabidopsis Atringla Atringlb and Atbmila-1 $A t b m i l b$ plants the lack of the RINGla-b or BMII $a-b$ genes expression, respectively, results in the initiation of embryogenic callus and embryo-like structures on various region of the plant, including cotyledons, shoot apex and leaves. These mutants also showed an enhanced expression of the genes associated with somatic embryogenesis, e.g., $B B M$, FUSCA3 (FUS3), LEC1, STM and WUS (Bratzel et al. 2010; Chen et al. 2010; Yang et al. 2013). In our study, however, the MtBMII expression in both lines during IP was almost identical. These results may indicate that BMI1 and RING1 are necessary to repress the embryogenic program after germination in Arabidopsis plants, but do not affect the genes mentioned above during induction of SE in M. truncatula. The next two genes, EMFI and VRNI encode non-sequence specific DNA-binding protein. Calonje et al. (2008) showed that Arabidopsis mutants with EMF1 loss-of-function generate the callus tissue and many developmental disorders. 
The MtEMF1 expression was higher in the M9-10a primary explants than in the M9 ones, but from day 2 of the SE induction phase its level was identical in both lines. In contrast to the MtEMF1 expression profile, the MtVRN1 transcription level was different in the tissue of both lines. During the IP, expression of MtVRN1 gradually increased in the M9-10a line, what was related to embryo formation during the differentiation phase, whereas expression of MtVRNl in the M9 line was unchanged and embryos was not formed. A strong increase in the MtVRN1 expression during the first 7 days of the IP in the M9-10a line can indicate its participation in starting the embryogenic pathway.

Certain genes of the Trithorax group probably can play a role in acquisition of the ability to cell embryogenicity by the M9-10a line. During the induction phase from day 2 to the end of induction, a higher expression of the ATX2, ATX3.1 and ATX3.2 genes was observed in M9-10a explants, compared to the non-embryogenic line. Our earlier results on PRC2 (Orłowska et al. 2017) and all the data discussed here show the Polycomb (PCRI and PCR2) and Trithorax group $(\operatorname{Tr} x G)$ genes to be involved in the switch of the embryogenic program.

In this study, we observed also an early expression of the MtWUS gene; expression in both lines in the presence of $0.5 \mu \mathrm{M} 2,4-\mathrm{D}$ and $1 \mu \mathrm{M}$ zeatin were at its maximum on 2 day. Similarly to results obtained by Rose et al. (2013), there were no differences in the expression profile of the MtWUS between both lines, thus this gene cannot be regarded as an embryogenic marker. In turn, studies on RNAi by Chen et al. (2009) showed that the MtWUS expression is important for production of callus tissue and somatic embryos in the embryogenic 2HA line of M. truncatula. The WUS expression profile they obtained during the IP of somatic embryogenesis, which was different than that observed in this study, resulted probably from growth regulators being used (10 $\mu \mathrm{M}$ NAA and $4 \mu \mathrm{M}$ BAP); expression of WUS was induced within 2 days, peaked after 7 days (coincident with the induction of totipotent stem cells), and dropped sharply on day 14. Su et al. (2009) showed the correct WUS expression, controlled by an appropriate exogenous auxin level, to be crucial for the Arabidopsis somatic embryo formation. AtWUS was demonstrated to enhance the conversion of non-embryogenic cells to embryogenic ones during SE in Gossypium hirsutum (Zheng et al. 2014). All the results discussed above may indicate that WUS expression takes place early during SE, the expression profile being dependent on the composition of hormones and their concentration in the medium.

Different expression profiles in the M9 and M9-10a lines were observed also in MtWOX5. In contrast to MtWUS, the expression of the gene took place later, between day 2 and day 7 (when rapid growth began) in the embryogenic line and was maintained at a high level until the end of the induction period. In the non-embryogenic line, the maximum expression the gene was observed a week later, i.e., after 14 days.

As in MtWOX 5 expression in the M. truncatula embryogenic line, the expression of the MtSTM gene increased rapidly from day 2 , and was maintained at a high level between day 7 and 14 of induction. Moreover, the lack of $M t S T M$ expression during the entire IP (21 days) in the non-embriogenic line clearly indicates that this gene can be used as a marker of embryogenic competence in $M$. truncatula. As mentioned earlier, the increased in expression observed in Arabidopsis lines with overexpression of Brassica STM, compared to transcription level in the wild-type line, is indicative of the gene's participation in somatic embryogenesis (Elhiti et al. 2010). We showed, for the first time, that during the early events in the SE induction, MtWUS is the first to be expressed, and transcription of MtSTM occurs only later, which confirms earlier data on zygotic embryogenesis in Arabidopsis (Mayer et al. 1998; Boscá et al. 2011). The WUS expression in zygotic embryos was detected at the early 16-cell embryo stage, the STM expression being observed at a late stage where the cotyledons arise.

Our results showed that, among the three genes tested (MtWUS, MtWOX5, MtSTM) during the IP in M. truncatula M9 and M9-10a lines, two of them, MtWUS and MtWOX5, can serve as markers of cell dedifferentiation in leaf explants of both lines, and only MtSTM may serve as a marker of embryogenic competence. $\mathrm{Xu}$ and Shen (2008) showed that the loss of function of RINGla and RINGIb genes induced expression of the Class I KNOX genes, including $S T M$, but did not affect the WUS transcription level in Arabidopsis leaves. Similarly, in somatic embryogenesis, a lower expression of MtRING1 in primary explants of the embryogenic line resulted in an increased MtSTM expression. MtSTM was not expressed in the non-embryogenic line showing a higher MtRINGI transcription.

In summary, expression of the $P R C 1$ and $\operatorname{Tr} x G$ proteincoding genes in primary leaf explants was lower in the embryogenic than in the non-embryogenic line, which may suggest that primary explants of the two lines differ in their chromatin methylation level. During the early events of the induction phase, the expression of the PRCI and $\operatorname{Tr} x G$ genes overlapped with higher transcript levels of MtWUS, MtWOX5 and MtSTM. Probably, the transcription level of MtWUS, MtWOX5 and MtSTM during the SE induction phase in $M$. truncatula may be a result of different proportions between the inhibiting (H3K27me3) and activating (H3K4me3) markers on these genes. This hypothesis, however, requires further study. 
Acknowledgements This work was partially supported by the National Scientific Centre (NCN) Grant No. NN303801340. The authors are very grateful to Professor Jan Kępczyński (Department of Plant Physiology and Genetic Engineering) for valuable advise during preparation of the manuscript and to Professor Lidia Skuza for making available the growth chambers of the Centre of Molecular Biology and Biotechnology, University of Szczecin. We are indebted to Teresa Radziejewska for linguistic assistance.

Author contributions EK and AO conceived and designed the research. $\mathrm{AO}$ constructed the experiments. $\mathrm{EK}$ and $\mathrm{AO}$ analyzed the data and wrote the manuscript. EK provided the funding.

\section{Compliance with ethical standards}

Conflict of interest The authors declare that they have not conflict of interest.

Open Access This article is distributed under the terms of the Creative Commons Attribution 4.0 International License (http://creativeco mmons.org/licenses/by/4.0/), which permits unrestricted use, distribution, and reproduction in any medium, provided you give appropriate credit to the original author(s) and the source, provide a link to the Creative Commons license, and indicate if changes were made.

\section{References}

Alvarez-Venegas R, Avramova Z (2012) Evolution of the PWWPdomain encoding genes in the plant and animal lineages. BMC Evol Bol 12:101

Alvarez-Venegas R, Pien S, Sadder M et al (2003) ATX-1, an Arabidopsis homolog of Trithorax activates flower homeotic genes. Curr Biol 13(8):627-637

Arroyo-Herrera A, Gonzalez AK, Moo RC et al (2008) Expression of WUSCHEL in Coffea canephora causes ectopic morphogenesis and increases somatic embryogenesis. Plant Cell Tiss Org 94(2): 171-180

Boscá S, Knauer S, Laux T (2011) Embryonic development in Arabidopsis thaliana: from the zygote division to the shoot meristem. Front Plant Sci 2:93

Bratzel F, López-Torrejón G, Koch M et al (2010) Keeping cell identity in Arabidopsis requires PRC1 RING-finger homologs that catalyze H2A monoubiquitination. Curr Biol 20(20):1853-1859

Calonje M (2014) PRC1 marks the difference in plant PcG repression. Mol Plant 7(3):459-471

Calonje M, Sanchez R, Chen L, Sung ZR (2008) EMBRYONIC FLOWER1 participates in Polycomb group-mediated AG gene silencing in Arabidopsis. Plant Cell 20(2):277-291

Chen SK, Kurdyukov S, Kereszt A et al (2009) The associated of homeobox gene expression with stem cell formation and morphogenesis in cultured Medicago truncatula. Planta 230(4):827-840

Chen D, Molitor A, Liu C, Shen WH (2010) The Arabidopsis PRC1like ring-finger proteins are necessary for repression of embryogenic traits during vegetative growth. Cell Res 20(12):1332-1344

Chen LQ, Luo JH, Cui ZH et al (2017) ATX3, ATX4, and ATX5 encode putative $\mathrm{H} 3 \mathrm{~K} 4$ methyltransferases and are critical for plant development. Plant Physiol 174(3):1795-1806

de la Paz Sanchez M, Aceves-García P, Petrone E et al (2015) The impact of Polycomb group (PcG) and Trithorax group (TrxG) epigenetic factors in plant plasticity. New Phytol 208(3):684-694
De-la-Peña C, Nic-Can GI, Galaz-Ávalos RM et al (2015) The role of chromatin modifications in somatic embryogenesis in plants. Front Plant Sci 6:635

Elhiti M, Tahir M, Gulden RH et al (2010) Modulation of embryoforming capacity in culture trough the expression of Brassica genes involved in the regulation of the shoot apical meristem. J Exp Bot 61(14):4069-4085

Endrizzi K, Moussian B, Haecker A et al (1996) The SHOOT MERISTEMLESS gene is required for maintenance of undifferentiated cells in Arabidopsis shoot and floral meristems and acts at a different regulatory level than the meristem genes WUSCHEL and ZWILLE. Plant J 10(6):967-979

Fehér A, Pasternak TP, Dudits D (2003) Transition of somatic plant cells to an embryogenic state. Plant Cell Tiss Org 74:201-228

Gro $\beta$-Hardt R, Lenhard M, Laux T (2002) WUSCHEL signaling functions in interregional communication during Arabidopsis ovule development. Genes Dev 16(9):1129-1138

He C, Chen X, Huang H, Lin X (2012) Reprogramming of $\mathrm{H} 3 \mathrm{~K} 27 \mathrm{me} 3$ is critical for acquisition of pluripotency from cultured Arabidopsis tissues. PLoS Genet 8:e1002911

Hennig L, Derkacheva M (2009) Diversity of Polycomb group complexes in plants: same rules, different players? Trends Genet 25(9):414-423

Igielski R, Kępczyńska E (2017) Gene expression and metabolite profiling of gibberellin biosynthesis during induction of somatic embryogenesis in Medicago truncatula Gaertn. PLoS ONE 12(7): 0182055

Jaudal M, Zhang L, Che C et al (2016) MtVRN2 is a Polycomb $V R N 2$-like gene which represses the transition to flowering in the model legume Medicago truncatula. Plant J 86(2):145-160

Kearse M, Moir R, Wilson A et al (2012) Geneious basic: an integrated and extendable desktop software platform for the organization and analysis of sequence data. Bioinformatics 28(12):1647-1649

King GJ, Chanson AH, McCallum EJ et al (2013) The Arabidopsis B3 domain protein VERNALIZATION1 (VRN1) is involved in processes essential for development, with structural and mutational studies revealing its DNA-binding surface. J Biol Chem 288(5):3198-3207

Koltai H, Dhandaydham M, Opperman C et al (2001) Overlapping plant signal transduction pathways induced by a parasitic nematode and a rhizobial endosymbiont. Mol Plant Microbe Interact 4(10):1168-1177

Kurdyukov S, Song Y, Sheahan MB, Rose RJ (2014) Transcriptional regulation of early embryo development in the model legume Medicago truncatula. Plant Cell Rep 33:349-362

Larsson AS, Landberg K, Meeks-Wagner DR (1998) The TERMINAL FLOWER2 (TFL2) gene controls the reproductive transition and meristem identity in Arabidopsis thaliana. Genetics 149(2):597-605

Ledwoń A, Gaj MD (2011) LEAFY COTYLEDON1, FUSCA3 expression and auxin treatment in relation to somatic embryogenesis induction in Arabidopsis. Plant Growth Regul 65:157-167

Livak KJ, Schmittgen TD (2001) Analysis of relative gene expression data using real-time quantitative PCR and the 2(-Delta Delta C(T)) Method. Methods 25:402-408

Long JA, Moan EI, Medford JI, Barton MK (1996) A member of the KNOTTED class of homeodomain proteins encoded by the STM gene of Arabidopsis. Nature 379(6560):66-69

Lotan T, Ohto M, Yee KM et al (1998) Arabidopsis LEAFY COTYLEDON1 is sufficient to induce embryo development in vegetative cells. Cell 93:1195-1205

Luerssen H, Kirik V, Herrmann P, Miséra S (1998) FUSCA3 encodes a protein with a conserved VP1/AB13-like B3 domain which is of functional importance for the regulation of seed maturation in Arabidopsis thaliana. Plant J 15(6):755-764 
Ma H, McMullen MD, Finer JJ (1994) Identification of a homeoboxcontaining gene with enhanced expression during soybean (Glycine max L.) somatic embryo development. Plant Mol Biol 24(3):465-473

Mayer KF, Schoof H, Haecker A et al (1998) Role of WUSCHEL in regulating stem cell fate in the Arabidopsis shoot meristem. Cell 95(6):805-815

Mozgová I, Muñoz-Viana R, Hennig L (2017) PRC2 represses hormone-induced somatic embryogenesis in vegetative tissue of Arabidopsis thaliana. PLoS Genet 13(1):e1006562

Ng DW, Wang T, Chandrasekharan MB et al (2007) Plant SET domaincontaining proteins: structure, function and regulation. Biochim Biophys Acta 1769(5-6):316-329

Nolan KE, Kurdyukov S, Rose RJ (2009) Expression of the SOMATIC EMBRYOGENESIS RECEPTOR-LIKE KINASE1 (SERK1) gene is associated with developmental change in the life cycle of the model legume Medicago truncatula. J Exp Bot 60(6):1759-1771

Orłowska A, Igielski R, Łagowska K, Kępczyńska E (2017) Identification of LEC1. L1L and Polycomb Repressive Complex 2 genes and their expression during the induction phase of Medicago truncatula Gaertn. somatic embryogenesis. Plant Cell Tissue Org. https ://doi.org/10.1007/s11240-016-1161-8

Reynolds TL (1997) Pollen embryogenesis. Plant Mol Biol 33(1):1-10

Rose JR, Sheahen MB, Tiew TW-Y (2013) Connecting stress to development in the induction of somatic embryogenesis. In: Aslam J, Srivastava PS, Sharma MP (eds) Somatic embryogenesis and gene expression, 1st edn. Narosa Publishing House, New Delhi, pp 146-156

Sanchez-Pulido L, Devos D, Sung ZR, Calonje M (2008) RAWUL: a new ubiquitin-like domain in PRC1 Ring finger proteins that unveils putative plant and worm PRC1 orthologs. BMC Genom 9:308

Sarkar AK, Luijten M, Miyashima S et al (2007) Conserved factors regulate signaling in Arabidopsis thaliana shoot and root steam cell organizers. Nature 446(7137):811-814

Schmidt ED, Guzzo F, Toonen MA, de Vries SC (1997) A leucine-rich repeat containing receptor-like kinase marks somatic plant cells component to form embryos. Development 124(10):2049-2062
Schuettengruber B, Martinez AM, Iovino N, Cavalli G (2011) Trithorax group proteins: switching genes on and keeping them active. Nat Rev Mol Cell Biol 12(12):799-814

Scofield S, Dewitte W, Murray JAH (2014) STM sustains stem cell function in the Arabidopsis shoot apical meristem and controls KNOX gene expression independently of the transcriptional repressor AS1. Plant Signal Behav 9:e28934

Smertenko A, Bozhkov PV (2014) Somatic embryogenesis: life and death processes during apical-basal pattering. J Exp Bot 65:1343-1360

Springer NM, Napoli CA, Selinger DA et al (2003) Comparative analysis of SET domain proteins in maize and Arabidopsis reveals multiple duplications preceding the divergence of monocots and dicots. Plant Physiol 132(2):907-925

Su YH, Zhao XY, Liu YB et al (2009) Auxin-induced WUS expression is essential for embryonic stem cell renewal during somatic embryogenesis in Arabidopsis. Plant J 59(3):448-460

Takatsuji H (1998) Zinc-finger transcription factors in plants. CMLS 54:582-596

Turck F, Roudier F, Farrona S et al (2007) Arabidopsis TFL2/LHP1 specifically associates with genes marked by trimethylation of histone H3 lysine 27. PLoS Genet 3(6): e86

$\mathrm{Xu}$ L, Shen WH (2008) Polycomb silencing of KNOX genes confines shoot stem cell niches in Arabidopsis. Curr Biol 23(18):1966-1971

Yang HY, Zhou C (1982) In vitro induction of haploid plants from unpollinated ovaries and ovules. Theor Appl Genet 63(2):97-104

Yang C, Bratzel F, Hohmann N et al (2013) VAL- and AtBMI1-mediated H2Aub initiate the switch from embryonic to postgerminative growth in Arabidopsis. Curr Biol 23(14):1324-1329

Zheng W, Zhang X, Yang Z et al (2014) AtWUSCHEL promotes formation of the embryogenic callus in Gossypium hirsutum. PLoS ONE 9(1):e87502

Zimmerman JL (1993) Somatic embryogenesis: a model for early development in higher plants. Plant Cell 5:1411-1423

Zuo J, Niu QW, Frugis G, Chua NH (2002) The WUSCHEL gene promotes vegetative-to-embryonic transition in Arabidopsis. Plant $\mathrm{J}$ 30(3):349-359 